\title{
Using Envelope Analysis and Compressive Sensing Method for Intelligent Fault Diagnosis of Ball Bearing
}

\author{
Khaldoon Fadhel Brethee*, Ghalib Rzayyig Ibrahim, Rashaq Abdullah Mohammed \\ Mechanical Engineering Department, Engineering College, University of Anbar, Al-Anbar, 10081, Iraq
}

\author{
A R T I C L E IN F O \\ Article history: \\ Received: 30 July, 2020 \\ Accepted: 11 September, 2020 \\ Online: 17 September, 2020
}

Keywords:

Ball bearing

Fault diagnosis

Vibration signal

Big data acquisition

Compressive sensing

\begin{abstract}
A B S T R A C T
Bearings are the key components of many rotating machines, in which serious failure or even major breakdown may occur due to their abnormal operation and defects. Thus, accurate fault diagnoses of bearing elements are essential for proactive predictive maintenance. However, the using of multiple sensors with high sampling rate reveal considerable shortages in the analysis of big data acquisition. Therefore, compressive sensing (CS) proposes in this study to overcome the aforementioned problems and support the fault diagnostic approach of ball bearing defects. The amount of data processed by CS technique can be significantly reduced to be more reliable for backup data. It can be a collaborative reconstruction method to compress the sampling data size and reliably exploiting similar sparsity structure of the acquired signal. Little attention has been paid for practically used sparseness of the CS converted signal in early fault detection of defects in ball bearing. Envelope analysis and CS technique are employed on experimental vibration data for fault detection in inner race and outer race of ball bearing. The results show that the reconstructed CS signal can characterize reliable features for bearing fault detection with some limitations in the range of compression ratio (up to 40\%) and the selection of reconstructed sparse bandwidth. Hence, envelope analysis can provide optimal bandwidth to reconstruct the sparse modulated signal of ball bearing to overcome the limitation of the CS method.
\end{abstract}

\section{Introduction}

Ball bearing is widely used to support rotors in rotating machinery, in which its failure is one of the foremost causes of system breakdowns. The faults in bearing could inevitably cause serious failures or even catastrophic damages to the machinery. Fatigue fracture, flacking, crack and wear are the most common failure modes of bearing, which starts by spalling in the bearing raceway and becomes more severe over a period of time. This may result in extensive damage to the operating mechanism of the bearing and the system assembly. Proper operation of bearing can be achieved by careful handling and right usage operation. The lifespan is relatively long, and it may eventually fail due to overloading, which result in significant increase in noise and vibration. Therefore, it is important to monitor the condition of the rotating machine elements, which is often effective to indicate the condition of raised faults at early stages. However, the using of multi sensors with high rate data acquisition comprise considerable shortages in transferring, storage and processing the acquired data, which is a major challenge in the detection efficiency [1-2].

*Corresponding Author: Khaldoon F. Brethee, University of Anbar, Iraq,

khaldon77m@uoanbar.edu.iq

www.astesj.com

https://dx.doi.org/10.25046/aj050546
The signature of bearing fault is generally masked by noise and spread over a wide frequency band, which make difficulties in fault diagnosis. Thus, different techniques have been used in various studies to detect various bearing faults at the early stages [3]. This can help to avoid long-term breakdown of the machinery. Vibration analysis is an effective way for condition assessment and fault diagnostics of various mechanical equipment applications. It can be processed with wide diversity signal processing methods to give valuable information for early detection of the abnormality in the bearing structure [4]. Basically, time domain and frequency domain analyses are used for tracking machinery operating conditions. Hence, various studies aimed to detect and diagnose the existence of a localized defect in bearing by using efficient time and frequency domain methods. Envelope analysis or demodulation extraction is an effective technique used for the fault detection approach from the amplitude variation of modulated signal [5]. It is widely used for detecting harmonics from the spectrum of vibration signal based on high frequency resonance techniques [6]. The envelope analysis has increasingly used in monitoring the health condition of bearings due to its simplicity and strength [7]. However, making envelope analysis suitable for 
speed and load transients are constrained and the defect information is not obvious and the selection of optimal band is the most difficult task.

Online monitoring of machinery elements requires analyse of big data acquisition to simultaneously propose real time condition monitoring approach. The continuous data streams should be analysed without distortion or being affected by scalability, which may put a big challenge for integrating the predictive maintenance decision. The powerful analysis of big data provides reliable decision support to detect the abnormality within the system components and indicate its performance. However, sampling rate is the major limit for acquiring big data acquisition. To get a good balance between a big data acquisition and the limitations of sampling theory, compressive sensing (CS) is a new signal processing technique, which can be used in machine condition monitoring due to its ability to sample the signal below the Nyquist sampling rate $[2,8]$. CS is widely used in various applications such as medical imaging, seismic imaging, communications and networks [9-11]. It can reduce the big data by a down-sample strategy with preserving reliable extraction of fault features [12]. The compression approaches can help the measuring of wireless transmission data by reducing its volume, hence significant reduction in energy consumption of wireless communication can be achieved [13-14]. The CS method can overcome the problem with the sampling, storage, transmission, and processing of big data acquisition.

The detection of bearing's fault feature can be extracted from the sparse samples of the vibration signal based on reconstruction compressive sensing signal. The CS can improve the condition monitoring approaches to be smaller, cheaper and efficient for used power in wireless devices [13]. It has the capability to develop useful information for condition monitoring of roller bearing with high level of accuracy [6, 8, 15-17], However, little attention has been paid for providing good sparseness of the CS converted signal, and apply it in fault diagnosis of ball bearing defects.

This study uses envelope analysis and CS method for monitoring and fault diagnosis of ball bearing defects, based on frequency shift and envelope analysis. These techniques has performed on vibration signal that acquired from experimental test of a ball bearing presented in an induction motor. It is expected that the CS approach achieves better classification accuracy for the purposed fault diagnosis from down sampling of the acquisition data.

\section{Envelope Analysis}

This method is mainly used to extract the periodic excitation in vibration signal of machinery, which can be used to detect and diagnose various defects in bearing. It has the ability to extract the amplitude modulated of vibration signal due to fault symptoms based on selected band pass filter. Figurel shows the general steps of envelope analysis method for detecting process, which based on the following equations $[5,15-16]$ :

$$
X(f)=\int_{-\infty}^{\infty} x(t) e^{-j \omega t} d t
$$

$$
\begin{gathered}
x(n)=\frac{1}{N} \sum_{k=0}^{N-1} X(f) e^{-j \omega k n} \\
x_{e n v}(n)=\sqrt{x(n) x^{*}(n)} \\
X_{e n v}(k)=\sum_{k=0}^{N-1} x_{e n v}(n) e^{-j \omega k n}
\end{gathered}
$$

where, $\mathrm{X}(\mathrm{f})$ is the fast Fourier transform of the raw vibration signal $\mathrm{x}(\mathrm{t}) . \mathrm{x}(\mathrm{n})$ is the resampled signal based on the selected bandwidth with $N$ number of samples. The analysed envelope signal is $\mathrm{x}_{\mathrm{env}}$ and its envelope spectrum is $X_{\text {env }}$. The main advantage of the envelope analysis is reliable fault detection of wide frequency range within certain frequency bands, which gives high excitation related to the impact of fault frequency. However, the envelope analysis is often mixed with noise, which may add some difficulties for the effective extraction of fault features.

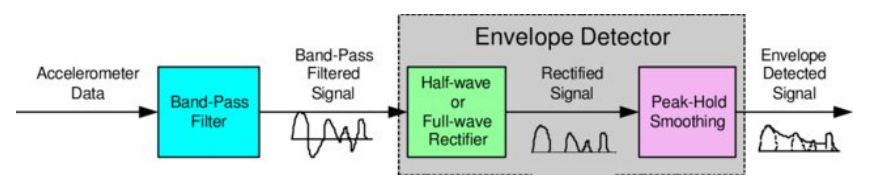

Figure 1: The general steps of envelope analysis method [18]

\section{Theory of Compressive Sensing}

In order to overcome the limitation of the sampling rate and processing of big data acquisition, compressive sensing method has been developed to resample the signal below the Nyquist sampling rate and express reliable sparse signal. The original signal, $\mathrm{x}(\mathrm{t})$ is reconstructed and multiplied by a sensing matrix to perform the compressed data, which denoted by $y$, as $[6,8-12]$ :

$$
y=\Phi x
$$

where, $\Phi$ is a random sensing matrix with size of $M \times N$. $M$ is the length of compressed signal $(y)$ with size of $M \leq N$.

The sparse components are produced with the dictionary of sparsifying transform $\Psi$ by the following equation:

$$
y=\Phi \Psi \eta
$$

The proposed CS strategy is used for defecting bearing's fault from the representation $\eta$ of the sparse signal $\mathrm{x}$, which can be demonstrated in figure 2. Before resampled the vibration signal with the bandwidth, the frequency was shifting with an exponential function, $e^{-j 2 \pi f t}$ based on interest shift frequency. The resampled signal is then divided into multiple partitions and averagely calculate root mean square (RMS) for each divided segment. The RMS values are composed based on the length of the sensing matrix $M(M<N)$ and reconstruct the signal with sparse representation, $\eta$ to determine its discrete Fourier transform. The extracted sparse representation of the signal can help to indicate the variation due to presence of defect. The defects existing in bearing elements are produced at a certain period and referred as bearing fault frequencies. Envelope demodulation technique is applied to the high-frequency band to extract the periodic 371 
excitation in the vibration signal and the harmonics of fault frequency components.

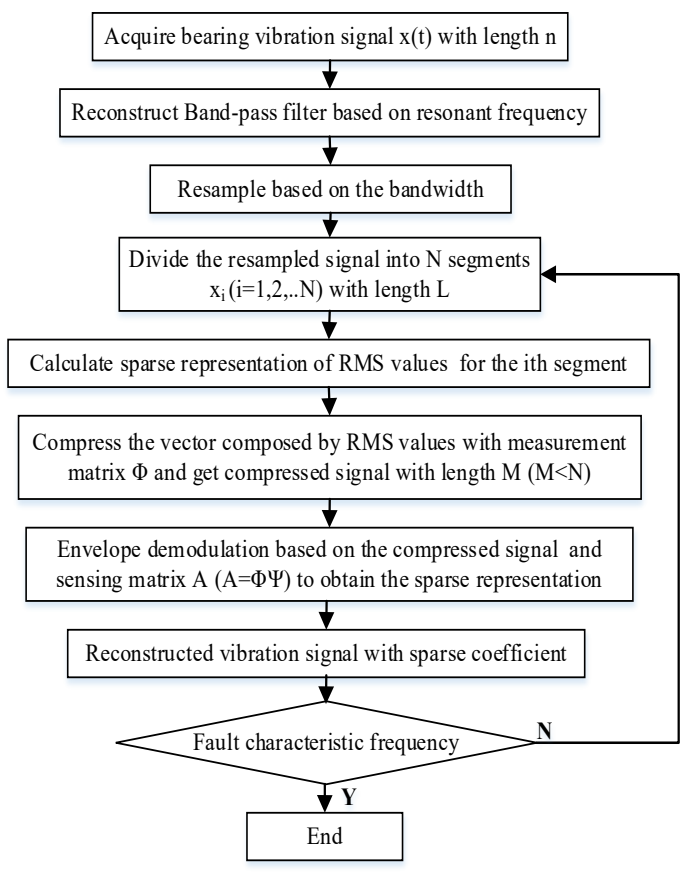

Figure 2: Fault detection strategy based on compressed sensing method

The determination of an optimal bandwidth to reconstruct the sparse signal of ball bearing imposes a severe challenge to apply the CS method. Due to modulation of the acquired vibration signal with the resonance frequency of the system or even with accelerometer, uncontrolled bandwidth could affect the performance analysis of the proposed method in a specific application. Hence, envelope analysis can give a clear indication to the effect of modulation within the measured vibration signal, which can help to overcome the limitation of the CS method.

\section{Experiment test}

Various experiments were performed with outer race and inner race faults in ball bearings to verify the effectiveness of using envelope analysis and the CS method. The experiment test was implemented in the laboratory to detect the seeded faults in ball bearings that used in an induction AC motor to drive a DC motor at various conditions. Figure 3 shows the construction of experiment test and the components of data acquisition system.

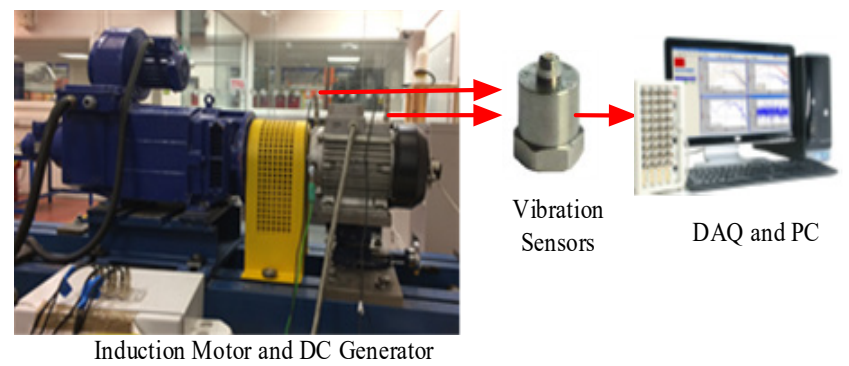

Figure 3: The Schematic diagram of experiment test system

In this experiment, three similar ball bearings have been used within the motor drive and their main operating parameters are detailed in Table 1.
Table 1: The description of ball bearing parameters

\begin{tabular}{|l|l|}
\hline Component & Value \\
\hline Pitch Diameter, $D_{p}$ & $46.4 \mathrm{~mm}$ \\
\hline Ball Diameter, $D_{b}$ & $9.53 \mathrm{~mm}$ \\
\hline Number of ball, $N_{p}$ & 9 \\
\hline Contact angle, $\beta$ & 0 \\
\hline $\begin{array}{l}\text { Operating motor speed } \\
\left.\text { (shaft frequency, } f_{r}\right)\end{array}$ & $1496 \mathrm{rpm}$ \\
& $(24.94 \mathrm{~Hz})$ \\
\hline
\end{tabular}

The three tested ball bearings are denoted as baseline (without fault) and the other two bearings are produced with inner race fault and outer race fault, respectively. The seeded bearing faults are shown in figure 4 . The failures of inner race and outer race of bearing are very common defects that could happen due to improper installation, incorrect lubrication, overloading, etc. Furthermore, the defect at any rotating element of machinery transmits to the bearing races and presents in terms of spalling, smearing, wear and surface distress. As a result, higher level of vibration is excited, which can be sensitive indicator to the starting of bearing deterioration.

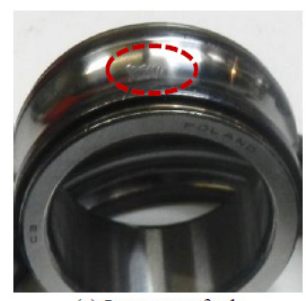

(a) Inner race fault

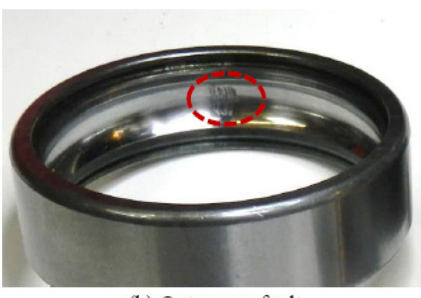

(b) Outer race fault
Figure 4: The Photographs of simulated ball bearing's faults

The ball bearing characterises with a complex excited vibration can be extracted from the measured vibration signal by an accelerometer mounted on the bearing's housing in vertical and horizontal directions. The excitation of bearing vibration is proportional with the structural design parameters of the ball bearing and the defects in their elements. The bearing vibration is rich in multi frequency components that induced in wide frequency bandwidth. The characteristic frequencies of the bearing defects can be identified by analysing the relative motion between the bearing elements and the rotor frequency, $f_{r}$. Then, the defect characteristic frequencies of the ball bearing elements can be indicated based on:

Outer race fault frequency: $f_{o}=\frac{N_{b}}{2} f_{r}\left(1-\frac{D_{b}}{D_{p}} \cos \beta\right)$

Inner race fault frequency: $f_{i}=\frac{N_{b}}{2} f_{r}\left(1+\frac{D_{b}}{D_{p}} \cos \beta\right)$

where $N_{p}$ is the number of rolled balls, $D_{b}$ is the ball diameter, $D_{p}$ is the pitch diameter of the bearing, and $\beta$ is the contact angle.

The seeded defects in the inner race and outer race can be detected based on their identified frequencies, and denoted by fault frequency are evaluated in Table 2 . 
Table 2: Fault frequency of ball bearing

\begin{tabular}{|l|l|}
\hline Parameter & Value (Hz) \\
\hline Inner Race Fault Frequency, $f_{i}$ & 135.26 \\
\hline Outer Race Fault Frequency, $f_{o}$ & 89.16 \\
\hline
\end{tabular}

\section{Vibration Data Analysis}

The vibration signal of ball bearing is mainly recorded in time domain for visual inspection, which is analysed by using advance signal processing methods. The analysis of vibration signal in time domain and frequency domain are obviously the two powerful techniques used for contemporary condition monitoring scheme. However, no clear impacts of the defects can be located and some frequency harmonics coincide and spread within wide waveform, and cause a lot of difficulties in the fault detection and diagnostic approaches [19]. Figure 5 and figure 6 show the time waveform and spectrum of vibration signal for three types of ball bearings (baseline (no fault), outer race fault and inner race fault).

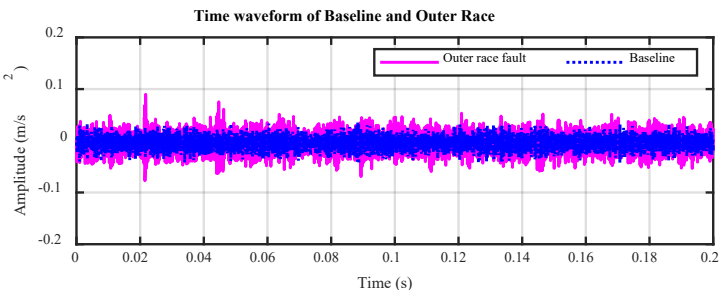

Spectrum of Baseline and Outer Race

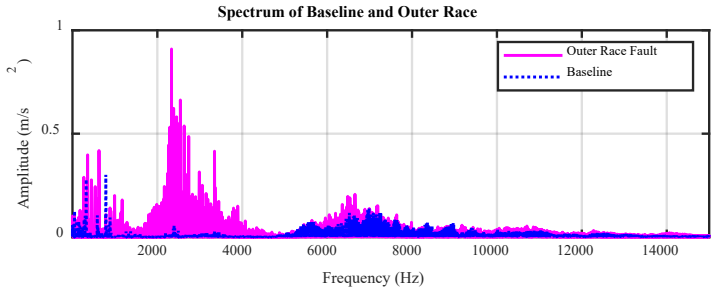

Figure 5: Vibration waveform and spectrum for baseline and outer race fault
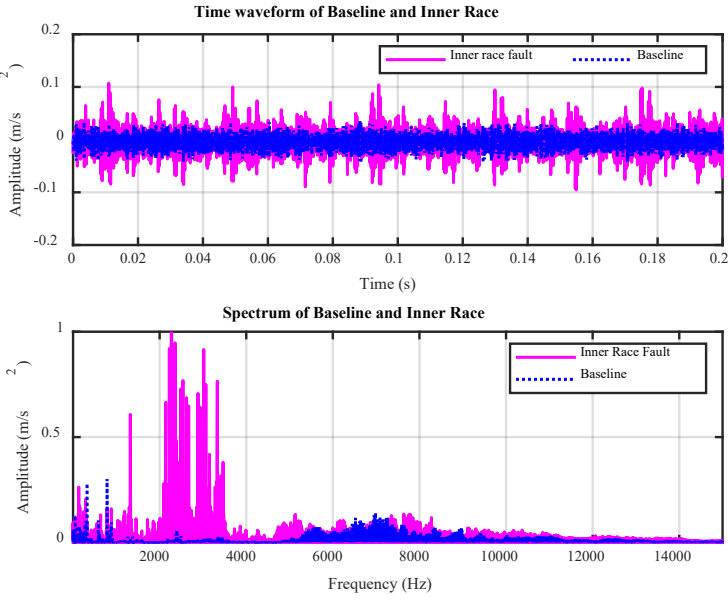

Figure 6: Vibration waveform and spectrum for baseline and inner race fault

The spectra of the bearings with outer race and inner race defects represents resonance around $3 \mathrm{kHz}$. This excitation effect is probably due to resonate the natural frequencies of bearing elements or the accelerometer with the periodic impacts of the bearing defects. Figure 7 shows the spectra of the tested bearings at low frequency band $(<500 \mathrm{~Hz})$ includes characteristic frequencies of the bearing defects. The difference in the bandwidth of the ball bearing defect is belonged to the variation of the transmission path of the excited vibration from the bearing components to the transducer. The vibration from the bearing with outer race defect has shorter path as compared with the inner race defect, where the harmonics cannot be revealed distinctively.

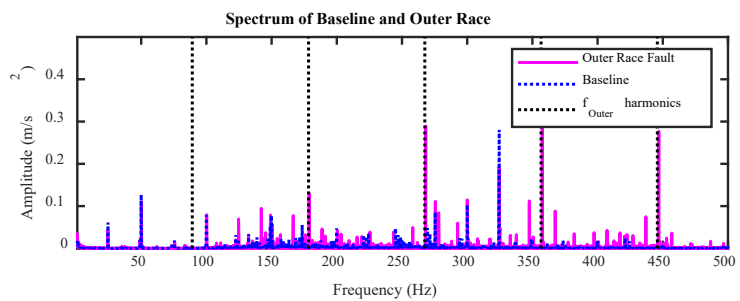

Spectrum of Baseline and Inner Race

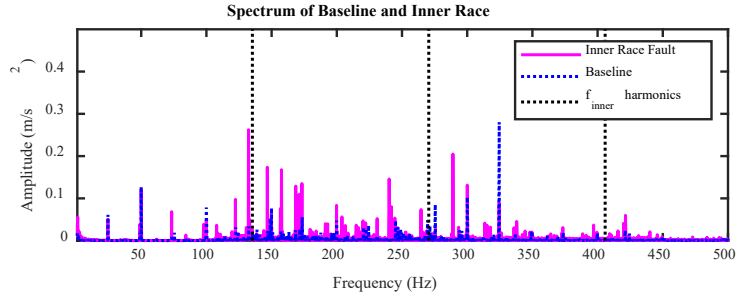

Figure 7: The spectra of the tested bearings at low frequency bands

\subsection{Envelope Analysis}

Amplitude modulation of vibration signal can be extracted from the envelope analysis in some specific bands. Figure 8 and figure 9 show the envelope analysis results of baseline, outer race fault and outer race fault bearings. The characteristic frequencies due to bearing defect and their harmonics $\left(f_{o}=89.16 \mathrm{~Hz}\right.$ and $f_{i}=135.26 \mathrm{~Hz}$ ) are significant, which are verified by using effective filter parameters. A band pass filter was used within the range of modulation bandwidth $(1 \mathrm{kHz}-5 \mathrm{kHz})$. This range was chosen based on the higher amplitude of spectra shown in figures 5 and 6 , where the bearing defects can be demodulated from the bearing natural frequencies. However, the determination of an optimal narrowband becomes poses a severe challenge for accurately employing envelope analysis.
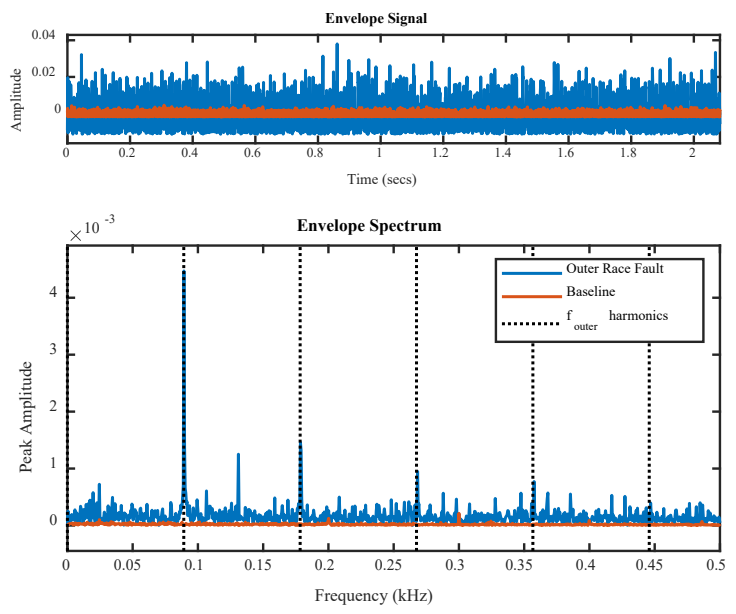

Figure 8: Envelope waveform and spectrum of baseline and outer race fault

\subsection{Compressive Sensing Analysis}

In order to overcome the problem with high sampling rate and big data acquisition, CS method can significantly reduce the 
consumption energy and the volume of transmission data [13-17]. The sampling rate can be reduced with using frequency shifting that can move the sparse signal from the high frequency range to a lower bandwidth. An exponential function $e^{-j 2 \pi f o t}$ was used to resample the ball bearing vibration signal with envelope resampling from the original sampling frequency $96 \mathrm{kHz}$ to $3 \mathrm{kHz}$. Then, divide the resampled signal into segments averagely and calculate the RMS value for every segment. The RMS values composed by all $N$ vector are compressed with a random sensing matrix to provide a reconstruct compressed signal with length $M$ $(M<N)$. The RMS value was determined for every 5 points, thus the sampling frequency is compressed to $600 \mathrm{~Hz}$ and the length of this vector is 2400 points and the CS process was repeated 10 times to acquire the average results of bearing faults, where random effects of measurement matrix can be reduced. Figure 10(a) shows the period of original RMS signal and the reconstructed RMS signal with CS for outer race bearing's fault, where the compression ratio $(M / N)$ of this process is up to $30 \%$.
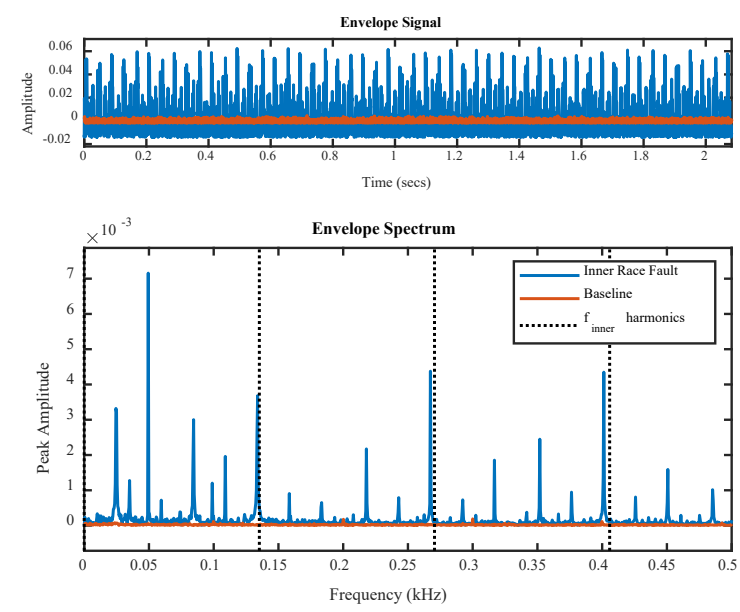

Figure 9: Envelope waveform and spectrum of baseline and inner race fault
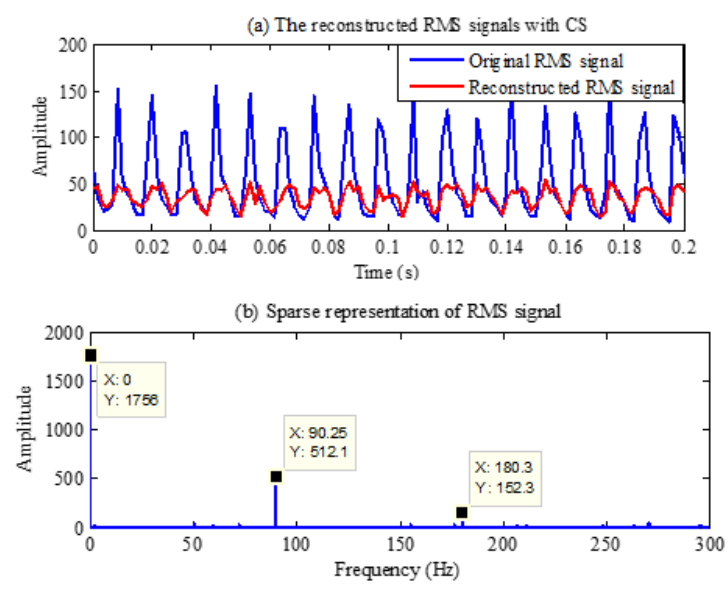

Figure 10: Reconstructed RMS signal and its sparse representation of vibration signal with outer race fault

It is clear that the amplitude of reconstructed signal is much smaller than that of the original RMS signal with maintaining good consistency so the fault frequency is not much affected, as demonstrated in figure 10(b). The sparse representation of the RMS signal is displayed at frequency $90.25 \mathrm{~Hz}$ and its harmonics, which are closer to the outer race fault frequency. The deviation in the fault frequency $f_{o}$ could result due to the variation in the rotor frequency $f_{r}$ of the induction motor.

Similarly, the trends of the reconstructed RMS signal for the ball bearing with inner race fault is shown in figure 11(a), where same parameters of averaging and compression ratio were used. The reconstructed RMS signal shows good consistent with the original RMS signal. Thus, the inner race fault frequency is displayed clearly at frequency $134.8 \mathrm{~Hz}$, as shown in figure 11(b). The frequency $50 \mathrm{~Hz}$ is the second harmonic frequency of the shaft rotating frequency, which is modulated with the inner race fault frequency. Therefore, it can be concluded that CS method can be reliably used to extract and detect the outer race and inner race faults of ball bearings and can be used to classify the defect according to the damaged element.
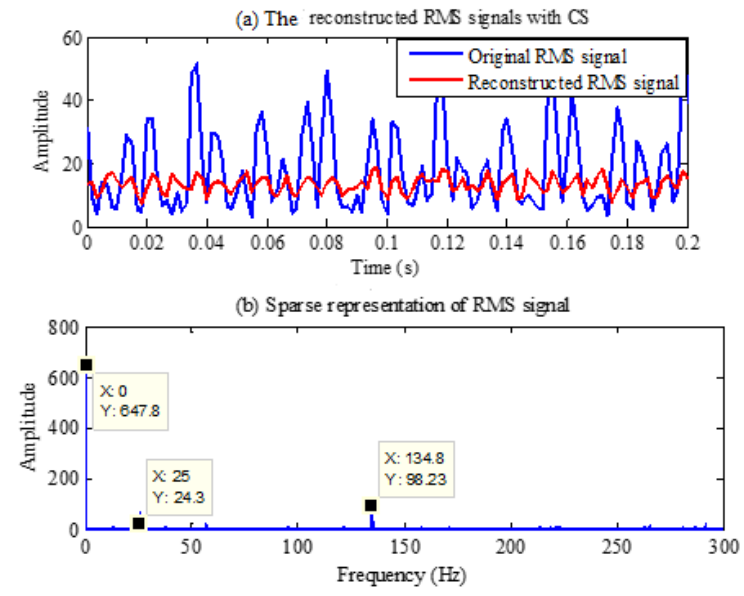

Figure 11: Reconstructed RMS signal and its sparse representation of vibration signal with inner race fault

The performance of the CS method was evaluated for various compression ratios $(M / N)$ by measuring signal to noise ratio (SNR) of the compressed signal. SNR is related to the reconstructed compressed signal and the original measured data. Figure 12 shows a clear decreasing in the SNR with increasing the $M / N$ ratio. It can be observed that the range of compression ratio between 30 $40 \%$ provides an acceptable level of SNR, which is significantly decreased for further compression of the signal.

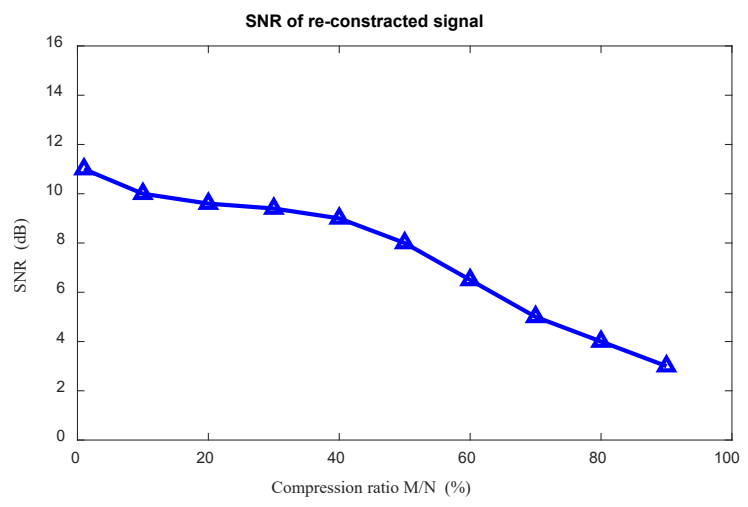

Figure 12: The evaluation of CS method for various compressions of the signal

Due to reduction in the sparse representation of the reconstructed signal, the diagnostic features of bearing defects are also influenced by the variation of the $M / N$ ratios. Figure 13 shows 
the variation in the amplitude peaks of the reconstructed sparse signal at the fault frequency features $\left(f_{o}\right.$ and $\left.f_{i}\right)$. The peaks of the fault frequency give clear indication for the sparse signal with the range of compression ratio up to $40 \%$. However, the amplitude of the fault frequencies is decreased for higher compression ratios, which may influence the fault diagnostic approaches. Good fault detection accuracy can be found for the compression ratio between $20-40 \%$, which can be evaluated based on the amplitude variation of bearing fault frequency. The higher accuracy of the CS method within the compression range was found for $M / N=30 \%$. The indication accuracy of bearing defects was found to be $94.7 \%$ for the $30 \%$ compressed signal as compared with the original signal. Moreover, the accuracy of the CS method was $91.8 \%$ for $40 \%$ compression ratio, which is relatively acceptable to reduce the data size and provide good indication of bearing defects.

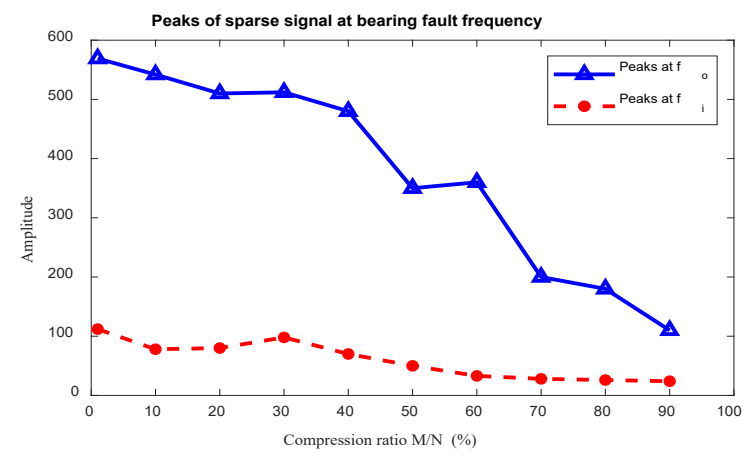

Figure 13: The amplitude peaks of the compressed signal at the diagnostic fault frequencies of inner race and outer race defects

\section{Conclusion}

This paper proposes the using of CS strategy as an approach for intelligent condition monitoring and fault detection of ball bearing. The CS method was applied based on frequency shifting of the high sparse response to a lower frequency band with applying envelope extraction at certain frequency bands, to overcome the problems of big data acquisition and the limitation of sampling theory. This process can help to significantly reduce the sampling rate with reliably extract the ball bearing's fault features, where sufficient information can be retrieved from the limited samples. Thereby, CS method can be reliably used to extract and detect ball bearing's faults, in which reliable fault detection can be extracted for compression ratio up to $40 \%$. The limitation of applicable CS method on the bearing fault diagnosis is the selection of suitable reconstructed sparse bandwidth. Hence, the bearing vibration signal is a modulated signal with bearing resonance frequencies or even with accelerometer elements, which can be extracted from envelope analysis. However, the determination of an optimal narrowband poses a severe challenge to be employed. More evaluation of the proposed method in terms of accuracy and cost comparison with other methods will be addressed in the future work with considering of wireless transmission data for a large range of applications.

\section{Conflict of Interest}

The authors declare no conflict of interest.

\section{Acknowledgment}

The authors would like to acknowledge the University of Anbar, Iraq and the University of Huddersfield, UK.

\section{References}

[1] L. Liu, "Robust fault detection and diagnosis for permanent magnet synchronous motors," PhD Thesis, College of Engineering, The Florida State University, USA, 2006

[2] M. Rani, S. Dhok, R. Deshmukh, "A Machine Condition Monitoring Framework Using Compressed Signal Processing," Sensors, 20(1), 319, 1-15, 2020, doi:10.3390/s20010319.

[3] N. Tandon, A. Choudhury, "A review of vibration and acoustic measurement methods for the detection of defects in rolling element bearings," Tribology international, 32(8), 469-480, 1999

[4] C. K. Mechefske, "Machine condition monitoring and fault diagnostics," In Vibration and Shock Handbook. Boca Raton, FL: CRC Press, Taylor and Francis Group, 25, 1-35, 2005.

[5] Y. T. Sheen, "An envelope analysis based on the resonance modes of the mechanical system for the bearing defect diagnosis," Measurement, 43(7), 912-934, 2010, doi:10.1016/j.measurement.2010.03.011.

[6] G. Tang, W. Hou, H. Wang, G. Luo, J. Ma, "Compressive sensing of roller bearing faults via harmonic detection from under-sampled vibration signals," Sensors, 15(10), 25648-25662, 2015, doi:10.3390/s151025648.

[7] D. Abboud, J. Antoni, S. Sieg-Zieba, M. Eltabach, "Envelope analysis of rotating machine vibrations in variable speed conditions: A comprehensive treatment," Mechanical Systems and Signal Processing, 84, 200-226, 2017, doi:10.1016/j.ymssp.2016.06.033.

[8] H. O. Ahmed, M. D. Wong, A. K. Nandi, "Intelligent condition monitoring method for bearing faults from highly compressed measurements using sparse over-complete features," Mechanical Systems and Signal Processing, 99, 459477, 2018, doi:10.1016/j.ymssp.2017.06.027.

[9] S. Li, L. Xu, X. Wang, "Compressed sensing signal and data acquisition in wireless sensor networks and internet of things," IEEE Transactions on Industrial Informatics, 9(4), 2177-2186, 2013, doi:10.1109/TII.2012.2189222.

[10] D. J. Holland, D. M. Malioutov, A. Blake, A. J. Sederman, L. F. Gladden, "Reducing data acquisition times in phase-encoded velocity imaging using compressed sensing," Journal of magnetic resonance, 203(2), 236-246, 2010, doi:10.1016/j.jmr.2010.01.001.

[11] S. Qaisar, R. M. Bilal, W. Iqbal, M. Naureen, S. Lee, "Compressive sensing: From theory to applications, a survey," Journal of Communications and networks, 15(5), 443-456, 2013, doi:10.1109/JCN.2013.000083.

[12] Y. Ma, X. Jia, H. Bai, G. Wang, G. Liu, C. Guo, "A new fault diagnosis method using deep belief network and compressive sensing," Journal of Vibroengineering, 22(1), 83-97, 2020, doi:10.21595/jve.2019.20850.

[13] J. Beuchert, F. Solowjow, S. Trimpe, T. Seel, "Overcoming Bandwidth Limitations in Wireless Sensor Networks by Exploitation of Cyclic Signal Patterns: An Event-triggered Learning Approach,” Sensors, 20(1), 260, 1-19, 2020, doi:10.3390/s20010260.

[14] L. Yin, C. Liu, S. Guo, Y. Yang, "Sparse random compressive sensing based data aggregation in wireless sensor networks," Concurrency and Computation: Practice and Experience, 32(3), e4455, 2020, doi: $10.1002 /$ cpe. 4455

[15] E. Sejdić, I. Orović, S. Stanković, "Compressive sensing meets timefrequency: an overview of recent advances in time-frequency processing of sparse signals," Digital signal processing, 77, 22-35, 2018, doi:10.1016/j.dsp.2017.07.016.

[16] X. F. Li, X. C. Fan, L. M. Jia, "Compressed sensing technology applied to fault diagnosis of train rolling bearing," In Applied Mechanics and Materials, Trans Tech Publications, 226, 2056-2061, 2012, doi:10.4028/www.scientific.net/AMM.226-228.2056.

[17] X. Tang, Y. Xu, F. Gu, A. D. Ball, G. Wang, "Fault detection of rolling element bearings using the frequency shift and envelope based compressive sensing," In 2017 23rd International Conference on Automation and Computing (ICAC), IEEE, 1-6, 2017, doi:10.23919/IConAC.2017.8082063.

[18] M. Lebold, K. McClintic, R. Campbell, C. Byington, K. Maynard, "Review of vibration analysis methods for gearbox diagnostics and prognostics," In Proceedings of the 54th meeting of the society for machinery failure prevention technology, Virginia Beach, VA, May 1-4, 623-634, 2000.

[19] H. Saruhan, S. Saridemir, A. Qicek, I. Uygur, "Vibration analysis of rolling element bearings defects," Journal of applied research and technology, 12(3), 384-395, 2014, doi:10.1016/S1665-6423(14)71620-7. 